\title{
Design and Evaluation of SunSpec-Compliant Smart Grid Controller with an Automated Hardware-in-the-Loop Testbed
}

\author{
Jay Johnson ${ }^{1}$ (D) $\cdot$ Ron Ablinger ${ }^{2} \cdot$ Roland Bründlinger $^{2} \cdot$ Bob Fox $^{3} \cdot$ Jack Flicker $^{4}$
}

Received: 25 April 2017 / Accepted: 23 August 2017 / Published online: 8 September 2017

(c) Springer Nature Singapore Pte Ltd. (outside the USA) 2017

\begin{abstract}
With increasing penetrations of inverter-based, renewable energy resources on electrical grids around the world, new distributed energy resource (DER) interconnection and interoperability requirements have been introduced to address emerging power system operator needs. The inverter-based power conversion systems are capable of communicating with grid operators, providing voltage and frequency support, and supporting the grid during faults. However, DER vendors are under pressure to quickly and reliably update the interoperability and electrical capabilities of their equipment for different jurisdictions with the rapidly changing landscape of disparate codes and standards. The necessary power hardware required for testing
\end{abstract}

Jay Johnson

jjohns2@sandia.gov

Ron Ablinger

Ron.Ablinger@ait.ac.at

Roland Bründlinger

Roland.Bruendlinger@ait.ac.at

Bob Fox

bob@sunspec.org

Jack Flicker

jdflick@sandia.gov

1 Photovoltaic and Distributed Systems Integration, Sandia National Laboratories, Albuquerque, NM, USA

2 Electric Energy Systems, AIT Austrian Institute of Technology, Vienna, Austria

3 SunSpec Alliance, San Jose, CA, USA

4 Advanced Microelectronics and Radiation Effects, Sandia National Laboratories, Albuquerque, NM, USA power systems under the wide variety of operational conditions may be untenable for many organizations. Therefore, we introduce an approach for the concurrent development of controls and application software through a controller hardware-in-the-loop (CHIL) testbed integrated with an automated testing platform that allows for the cost-effective, flexible evaluation of advanced grid support functions without the need for large and expensive power hardware. We show this CHIL capability through the demonstration and automation of interconnection tests with a $34.5 \mathrm{~kW}$ Austrian Institute of Technology (AIT) smart grid converter (SGC) connected to a Typhoon HIL system. We have demonstrated the CHIL system with regards to connect/disconnect, active power curtailment, fixed power factor, reactive power control, volt-var, and frequency-watt advanced grid functionality tests. For all tests, the automated CHIL testing protocols for advanced functions were sufficient to demonstrate and evaluate the grid support behavior of the equipment under test.

Keywords Systems integration · Grid integration · Inverter design · Advanced grid-support functions $\cdot$ Controller hardware-in-the-loop $\cdot$ DER interoperability

\section{Introduction}

Recently, distributed energy resource (DER) interconnection and interoperability codes and standards around the world are being revised to provide grid operators with tools for providing frequency, voltage, and protection services [1-3]. This rapid evolution is driven by grid operator needs as greater penetrations of distributed renewable energy resources are displacing traditional thermal generation. The widespread adoption and distributed nature of 
DER is causing voltage regulation challenges for distribution circuits [4]; greater frequency deviations due to reduced inertia in power systems [5]; and new protection challenges from fuse, relay, and circuit breaker desensitization [6]. In order to maintain a safe, stable power system, DERs must actively participate in grid operations.

DERs can provide grid services through both programmable autonomous functions as well as remotely commanded actions. Many of these functions were described in an EPRI report [7] and later formalized in IEC Technical Report 61850-90-7 [8] — soon to be standardized in IEC 61850-7-420 [9]. Many of these same functions-or variations on them-have been required in Europe [2, 3], and more recently in California and Hawaii in the U.S. In its recent full revision currently undergoing the final ballot phase, the U.S. national interconnection standard, IEEE Std. 1547 [10], added many of these functions and interoperability requirements. With the addition of these requirements, there are two emerging needs: (a) equipment vendors must redesign their equipment to meet new grid standards by systematically validating DER performance and (b) standards development organizations (SDOs) must establish certification protocols (e.g., IEEE 1547.1 [11] and UL 1741 [12]) to list equipment.

To address these near-term industry needs, Sandia National Laboratories, Austrian Institute of Technology (AIT), SunSpec Alliance, and Typhoon HIL are collaborating to introduce a new approach for rapid development of DER controls, and interoperability and interconnection protocols. This approach expands previous power systems research on DER grid-support function evaluations under the International Smart Grid Action Network (ISGAN) Smart Grid International Research Facility Network (SIRFN) [2, 13, 14] and integrates test automation software from a Sandia-SunSpec-SIRFN development project $[15,16]$ with the AIT Smart Grid Converter (SGC) development and controller hardware-in-the-loop (CHIL) research platform [17-19]. This methodology provides key benefits over traditional power laboratory testing, by:

- Validating smart inverter grid-support functions without expensive power laboratory equipment, using lowvoltage benchtop equipment,

- Executing certification tests to verify controller operation prior to hardware integration,

- Allowing quick design iterations of the communication system to provide interoperability for a range of standards [20], and

- Quickly refining SDO draft interconnection and interoperability codes and standards prior to publication.

CHIL methodology provides key benefits over traditional full-scale power laboratory testing by minimizing the power equipment required to evaluate the system while also evaluating control logic and interoperability interfaces prior to integration with hardware. By eliminating power requirements, the CHIL test setup has the flexibility to easily operate for a range of systems (e.g. single and threephase devices, different nameplate ratings, grid functions, and operating capabilities), control schemes, and grid conditions. CHIL has been demonstrated in a wide variety of DER utilized grid topologies, including Medium Voltage DC equipment [21], microgrids [22, 23], and smart grids [24] and is widely accepted as a cost-effective method to determine the behavior of power systems under a variety of grid operating conditions.

With high demand for certification lab time equipment and the short timelines for equipment listing, a highthroughput testing method is sorely needed for rapid deployment of smart grid technologies. Power hardware-in-loop (PHIL) has previously been used to determine PV inverter behavior to certification requirements [25] but CHIL is more slowly entering certification standards as an acceptable alternative to power system testing. Standards development organizations have recognized that it becomes increasingly difficult to test full-scale equipment as power electronics equipment continues to grow to megawatt-scale devices or networks of devices. The IEEE 2030.8 standard for microgrid controller testing [26] will likely include a CHIL testbed and a newly started IEEE P2004 standard [27] will include recommended practices for HIL testing of electric power apparatuses and controls. In January 2014, NREL released a test protocol for voltage and frequency trip and ride through experiments that allowed signal injection [28]. Signal injection is a type of CHIL experiment in which the power system is not perturbed, but rather the pertinent DER measurements are changed to reflect the grid conditions of the test. For instance, if a high voltage trip test needed to be completed on a $2 \mathrm{MW}$ inverter, a 2 MVA or larger grid simulator would not need to generate the high voltage test conditions, but rather the equivalent scaled DER voltage measurement signal would be injected into the controller to represent the high voltage condition. In the IEEE 1547 revision process [29], signal injection was discussed at length and in the balloted version it is allowable for type tests "provided that the completeness of the required test is not compromised." At this time, it is unclear how many DER vendors or Nationally Recognized Testing Laboratories (NRTLs) will use this technique; however, inclusion of this option indicates a shift in testing toward CHIL certification testbeds.

One final goal of this project is to provide institutions in emerging economies, universities, and utilities with a lowcost, low-voltage platform for conducting DER grid-support research. As emerging economies begin to experience rapid growth in power demand and integrate higher penetrations of renewables, there is a need to establish DER requirements 
for grid support functions. While laboratories in countries with a significant history in DER integration possess extensive testbeds for evaluating the performance of DER equipment with grid simulators, PV/battery simulators, data acquisition systems, meters, and communication infrastructure, in many cases, especially for emerging economies such as India, Mexico, and South Africa, expensive power systems testing and evaluation infrastructure for full-scale unit testing is limited. These countries require a means to quickly develop results-based interconnection standards and evaluate equipment to these requirements. Therefore, versatile and flexible testing methodologies are needed to understand system response without the risk and expense of full-scale power system evaluation. Using the CHIL benchtop system, universities, research laboratories, regulators, utilities, and equipment vendors could collaborate to develop comprehensive interconnection and interoperability standards for their country.

In this paper, the design and use of a smart inverter CHIL (Si-CHIL) platform consisting of an integrated SunSpeccompliant server, smart grid controller, CHIL hardware/software system, and automated test platform is presented. The testing methodology along with results of a number of advanced grid-support functions including connect/disconnect, active power curtailment, fixed power factor, reactive power, volt-var, and frequency-watt are provided.

\section{Laboratory Testbed Configuration}

The hardware setup used for the design and validation of the advanced smart grid converter is shown in Fig. 1.



Fig. 1 Si-CHIL test setup with Typhoon HIL 602 simulator and AIT SGC HIL Connect that integrates the ASGC components with a SunSpec/supervisory control and data acquisition (SCADA) graphical user interface (GUI)
The testbed consists of the real-time simulation system (Typhoon HIL 602) and simulation software, AIT SGC (sometimes shortened to ASGC), SunSpec Modbus server, and a Windows computer to automate the tests.

The HIL 602 unit provides a real-time $\mu$ s resolution simulation of the converter power stage, the AC power grid and the solar array and connects to the Smart Grid Converter Controller through analog and digital inputs and outputs (I/Os), representing grid voltages, currents and Insulated-Gate Bipolar Transistor (IGBT) Pulse Width Modulation (PWM) signals. The AIT SGC represents the grid-connected PV inverter providing a broad range of advanced grid support capabilities. The equipment under test (EUT) modes and settings-ASGC, in this case-were accessed through a dedicated communications processor running a SunSpec Modbus TCP server that handles the low-level communication with the ASGC via a secured binary protocol. Figure 2 shows the internal layout of the HIL Connect and the connectivity features. The AIT SGC HIL Connect is comprised of the SGC Controller, which is powered by a Real Time Operating System (RTOS) running on a Digital Signal Processor (DSP), and a FieldProgrammable Gate Array (FPGA) connected to the DSP via an External Memory Interface (EMIF) bus capable of Gbit/s cluster-synchronization for various values across a multiple SGC controllers via the Power Link. A valid SunSpec client request is processed by the communication processor and sent to the DSP. The DSP communicates via a low-level binary Inter-Processor Communication (IPC) protocol with the SunSpec Modbus server, an ARM-based communication processor.

To fully implement automated tests, the SunSpec System Validation Platform (SVP) was utilized as a central control platform. The SVP is a versatile automated certification platform that allows the test sequences to be scripted through abstraction layers to test equipment and EUTs using the Python programming language. The abstraction layers allow identical test logic to be executed for different test equipment/EUTs, e.g., with actual PV inverters or simulated inverters through the use of CHIL. In this case, the SVP is used to automate the testing sequence by issuing commands to adjust AC grid and PV parameters in the CHIL simulation environment, capture data from the simulation, and adjust the functional modes and settings in the EUT. The SVP communicates to the HIL simulator through a USB connection using the Typhoon HIL Application Program Interface (API) and EUT settings are changed via SunSpec Modbus TCP over Ethernet as shown in Fig. 3.

The EUT represents a $34.5 \mathrm{~kW}$ grid-tie photovoltaic inverter with full four-quadrant operation, capable of providing a broad range of advanced grid support capabilities via the AIT SGC controller. The SGC uses a propriety suite of algorithms for advanced inverter controls. The details 


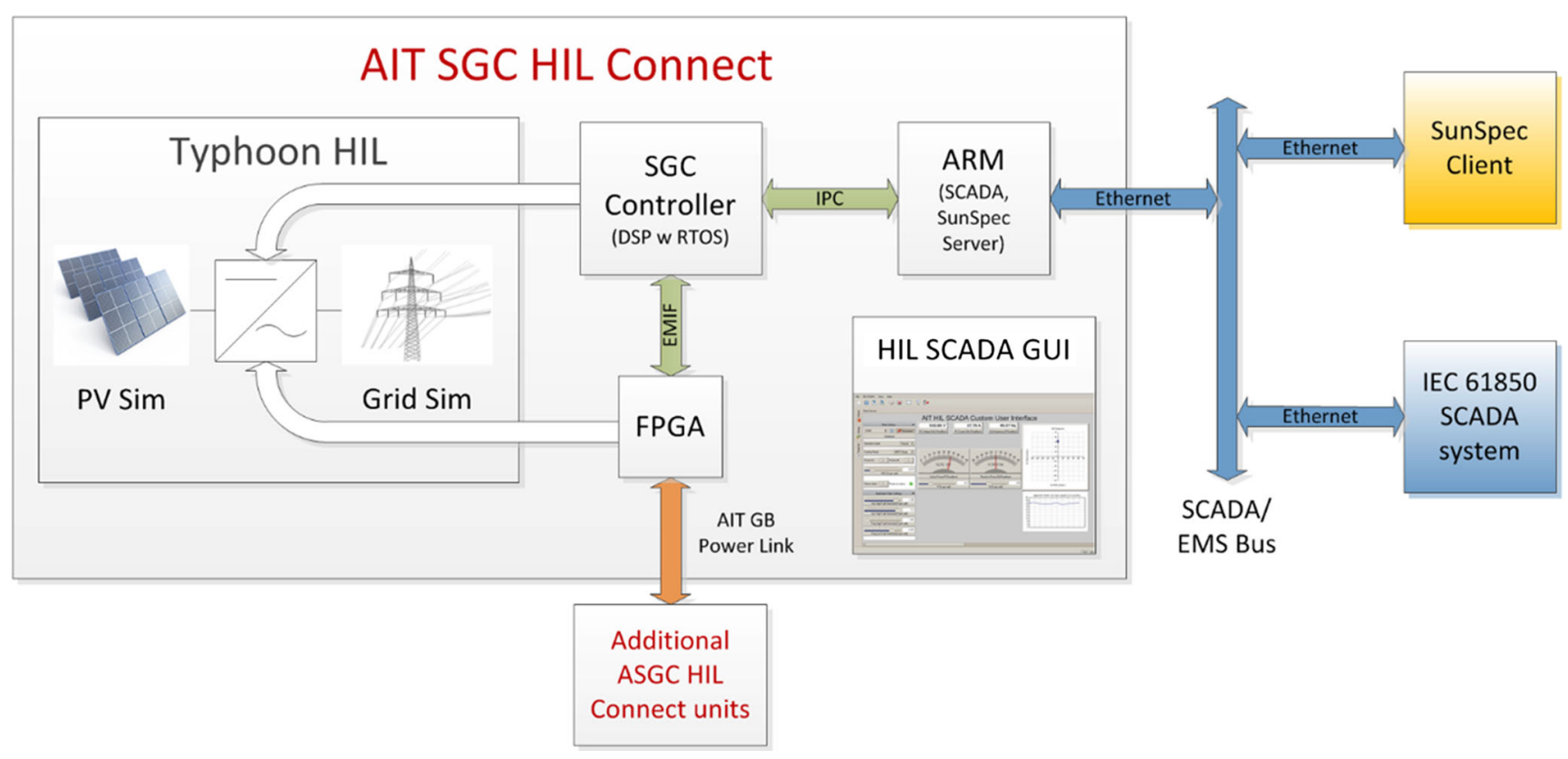

Fig. 2 Layout of the AIT SGC HIL Connect test system

of the algorithms, their capabilities, and disadvantages are not discussed here (but covered in [17-19]), as the purpose of the experimental setup described herein is to demonstrate the flexibility of evaluating a wide variety of control schemes and algorithms.
Although the EUT is capable of four-quadrant operation, in these tests, only positive active power operation was witnessed because and no storage was present. The DC power was provided by a simulated PV array with a maximum power point $\left(\mathrm{P}_{\mathrm{mp}}\right)$ of $36.24 \mathrm{~kW}$ at $1000 \mathrm{~W} / \mathrm{m}^{2}$. The

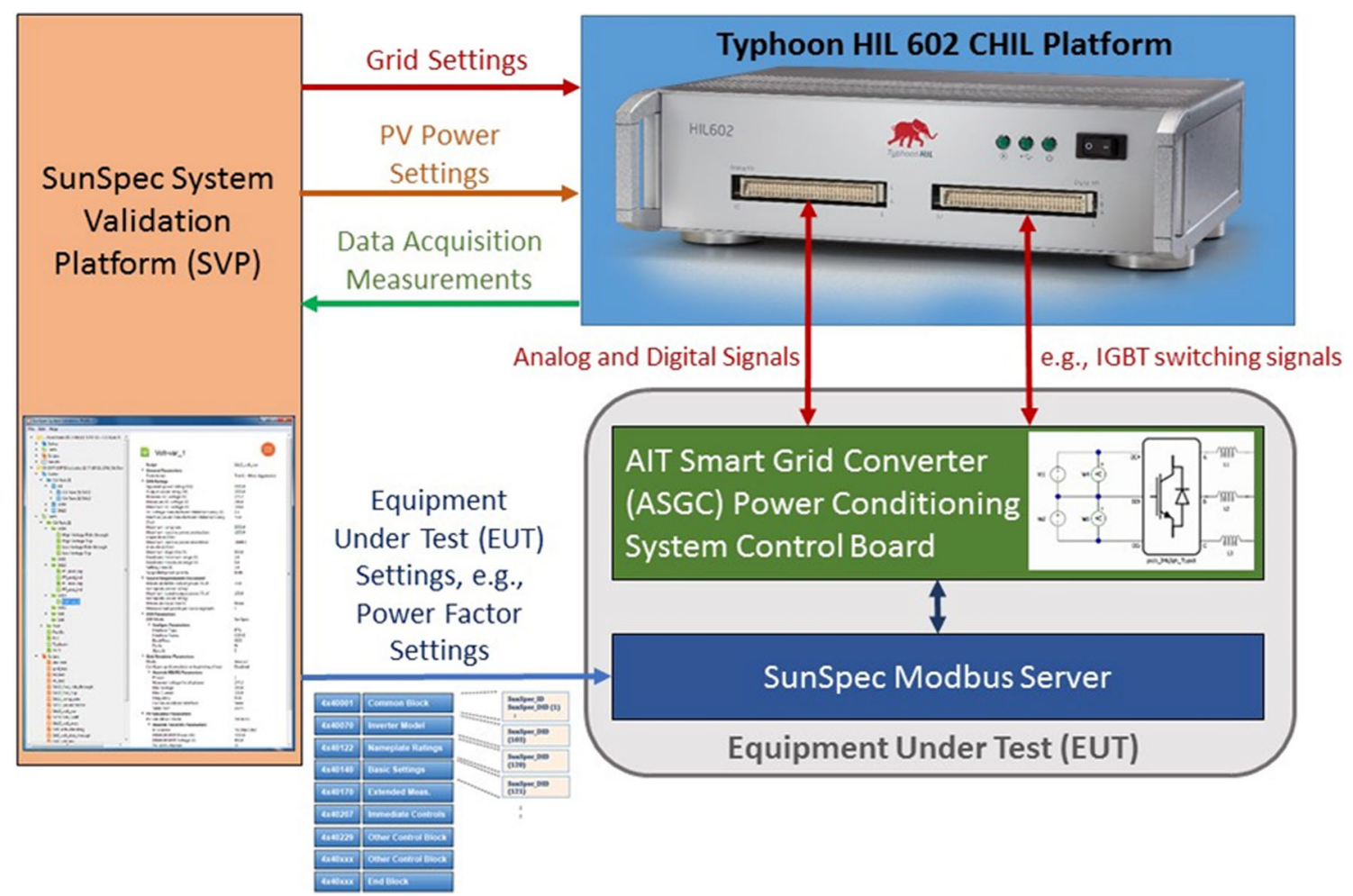

Fig. 3 Si-CHIL test configuration for automated grid-support function testing 
Fig. 4 EUT response from five disconnect and five connect commands

\section{Connect/Disconnect Response}



EUT response to different SunSpec settings was initially assessed using the Typhoon HIL Control Center and the SunSpec Dashboard [30] to determine the EUT functional limitations (e.g., minimum and maximum grid frequency and voltage) and available grid support functions. These limitations where observed when conducting the automated experiments.

\section{Experimental Results}

The Si-CHIL setup is capable of testing the EUT compliance to any number of grid codes and standards using the SVP. For this work, simple experiments were scripted to evaluate the grid-support capabilities of the EUT. Data was collected from the Typhoon HIL through the Typhoon HIL
Python API every $200 \mathrm{~ms}$ from analog signals representing the AC and DC current and voltage, and from calculated CHIL channels including root mean square (RMS) AC power, RMS reactive power, and power factor. In the experiments, the PV power was adjusted by setting the simulated PV irradiance level, and the grid voltage and frequency were changed for all three single-phase voltage sources simultaneously via the Typhoon API.

\section{Connect/Disconnect}

The connect/disconnect function (IEC 61850-90-7 INV1) isolates the DER from the grid. Typically, isolation is created by gate blocking the $\mathrm{H}$-bridge semiconductor switches, but it could also be accomplished by actuating a contactor to provide galvanic isolation.
Fig. 5 Active power of the EUT when operating in curtailment mode




To evaluate the EUT, five disconnect and five connect commands were issued to the Modbus Server at $15 \mathrm{~s}$ intervals. The results are shown in Fig. 4. As can be seen, the disconnect command was acted upon in less than $1 \mathrm{~s}$. However, the connect command took longer, $\sim 5 \mathrm{~s}$, while the EUT resynchronized with the grid and began to output power. Then the power increased for another $\sim 5 \mathrm{~s}$ as the maximum power point tracker (MPPT) moved to the PV $\mathrm{P}_{\mathrm{mp}}$.

\section{Active Power Curtailment}

The curtailment function (IEC 61850-90-7 INV2) is used to reduce the DER output historically mitigated voltage or frequency impacts [31] or ensure circuit protection, but it has the potential to provide bulk grid services. The PV irradiance was set to $1000 \mathrm{~W} / \mathrm{m}^{2}$ and EUT was commanded three times to change active power from $10 \%$ to $100 \%$ to $10 \%$ output at $10 \%$ increments. The settings were issued at $3 \mathrm{~s}$ intervals. As can be seen in Fig. 5, the EUT tracks the signal well except at the MPP where device efficiency prevented the DER from reaching nameplate power.

\section{Fixed Power Factor}

The fixed power factor (PF) function (IEC 61850-90-7 INV3), or $\cos (\phi)$ (assuming small distortion), is used to inject or absorb reactive power for voltage regulation. Power factors every $30^{\circ}$ were issued to the EUT while the PV

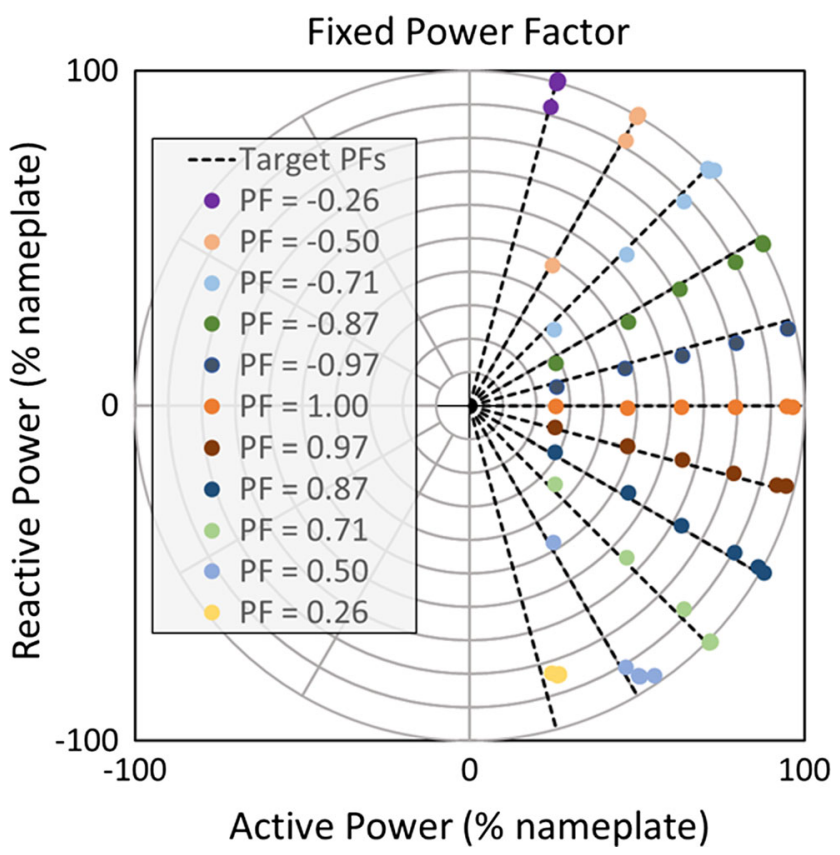

Fig. 6 Fixed power factor results for seven different PV irradiance levels

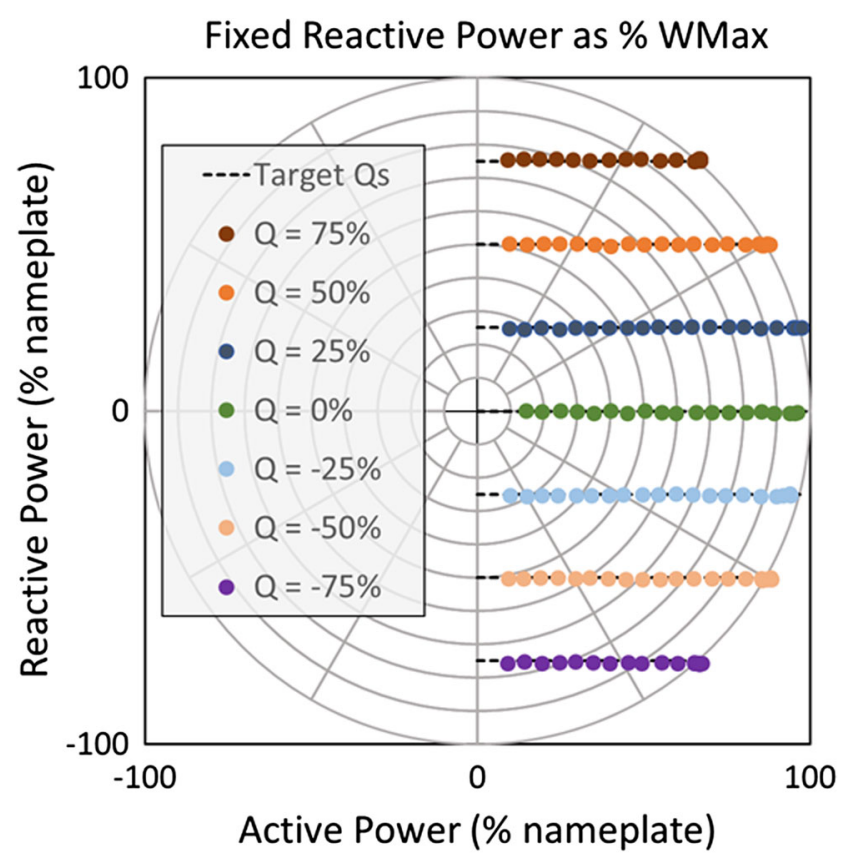

Fig. 7 Seven commanded reactive power settings (WMax)

irradiance was set to $100,250,450,600,750,900$, and $1000 \mathrm{~W} / \mathrm{m}^{2}$. After a $2 \mathrm{~s}$ settling time, the active and reactive power were recorded and plotted in Fig. 6 . As can be seen in the P-Q plane, the EUT accurately provided the PF setting while maximizing the active power output of the EUT and maintaining the apparent power limit of the device, represented by the outside circle in the figure. The PF accuracy of the EUT tends to decreases as power factor moves away



Fig. 8 Seven commanded reactive power settings (VArMax) 


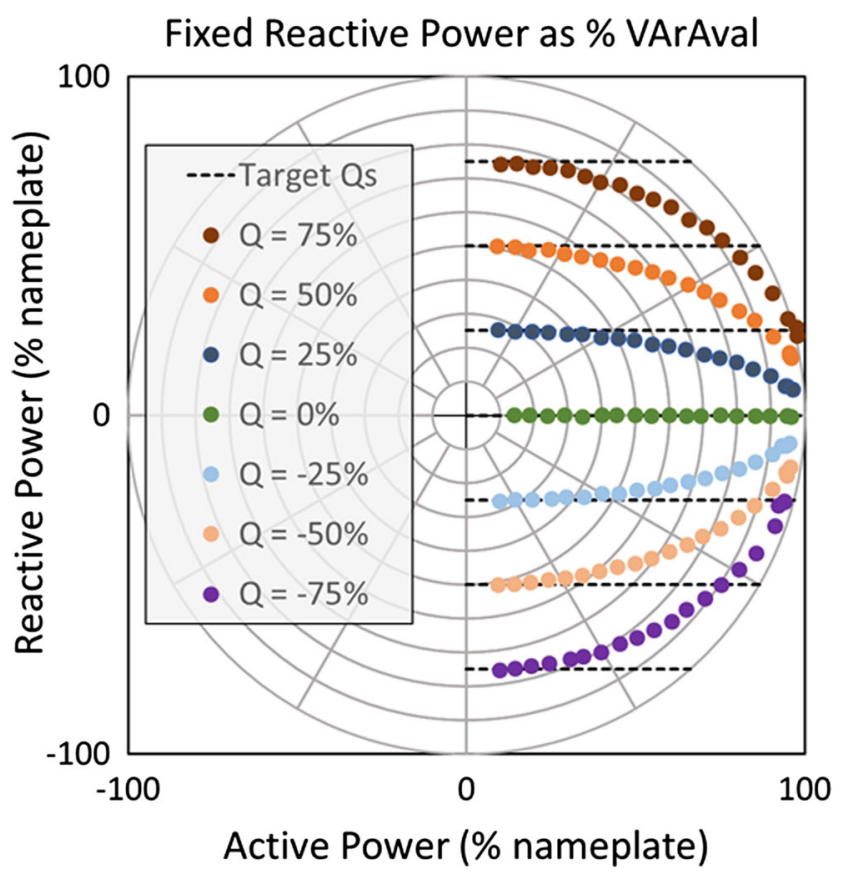

Fig. 9 Seven commanded reactive power settings (VArAval)

from unity because deviations in phase angle yield larger PF errors.

\section{Reactive Power}

Another voltage regulation function is the commanded reactive power mode. This function can set the reactive power in units of maximum active power (WMax), maximum reactive power (VArMax), or available reactive power
(VArAval)—where active power is not curtailed. The PV irradiance was set to 20 points between 100 and $1000 \mathrm{~W} / \mathrm{m}^{2}$ for seven reactive power settings. For this EUT, the WMax and VArMax values were both 34500 , so the results of these functions were very similar, as seen in Figs. 7 and 8. In the case of setting the reactive power as a function of $\% \mathrm{VAr}-$ Aval, the reactive power was reduced at higher irradiances to avoid active power curtailment, shown in Fig. 9.

\section{Volt-Var}

The volt-var (VV) function, or $\mathrm{Q}(\mathrm{V})$, is an autonomous DER mode that is designed for autonomous voltage regulation based on the local EUT grid voltage measurements. Two different VV curves were programmed into the EUT using the SunSpec parameters and tested at 100 voltage points for three different irradiance levels $\left(200,600\right.$, and $\left.1000 \mathrm{~W} / \mathrm{m}^{2}\right)$. There was close alignment of the EUT reactive power output and the target for the two VV curves, as shown in Figs. 10 and 11. The slight horizontal shift in the curve is thought to be due to calibration and measurement accuracy of the lab setup. Additional analysis of the VV curve for this devices is provided in [20].

\section{Frequency-Watt Function}

The frequency-watt, FW, or $\mathrm{P}(\mathrm{f})$ function is used to support bulk system balancing. The EUT autonomously changes its active power output based on local grid frequency measurements. For instance, in Germany and Austria, VDE-AR-N 4105 requires a $40 \% / \mathrm{Hz}$ reduction in $\mathrm{PV}$ power above $50.2 \mathrm{~Hz}$ [32]. Per IEC 61850-90-7 and SunSpec models,
Fig. 10 Volt-Var behavior of the EUT for Curve 1

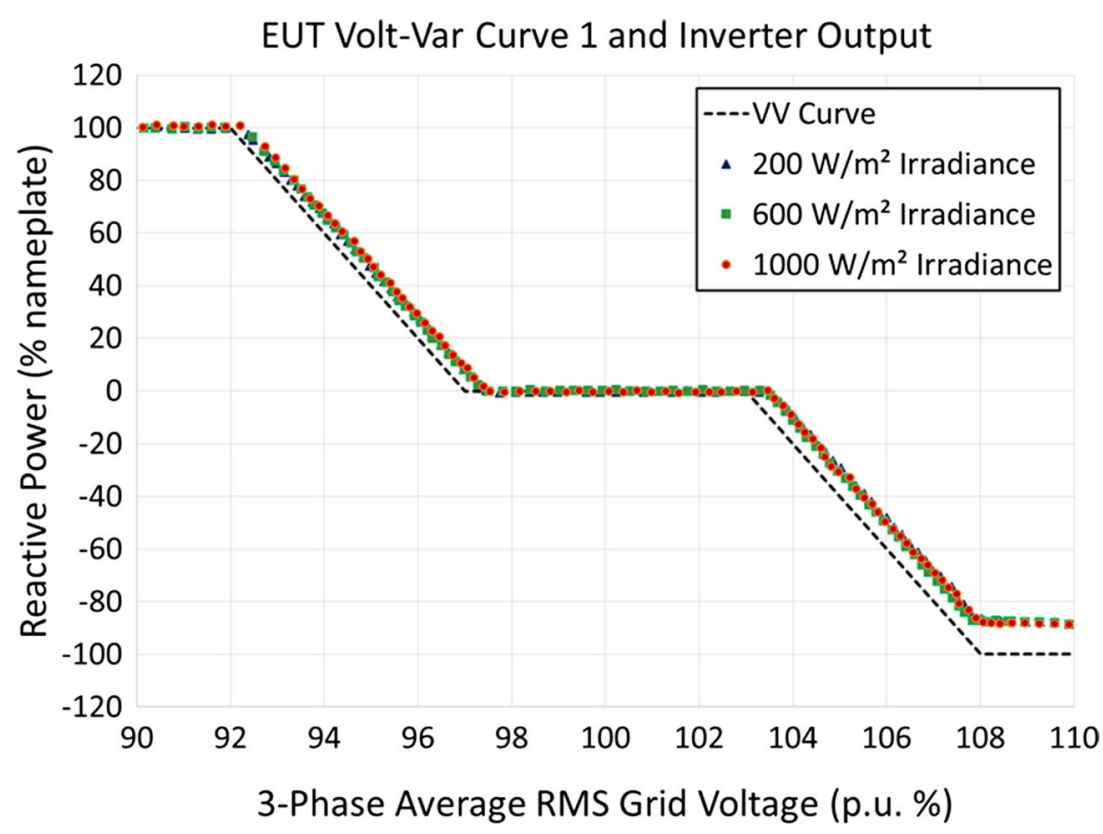


Fig. 11 Volt-Var behavior of the EUT for Curve 2

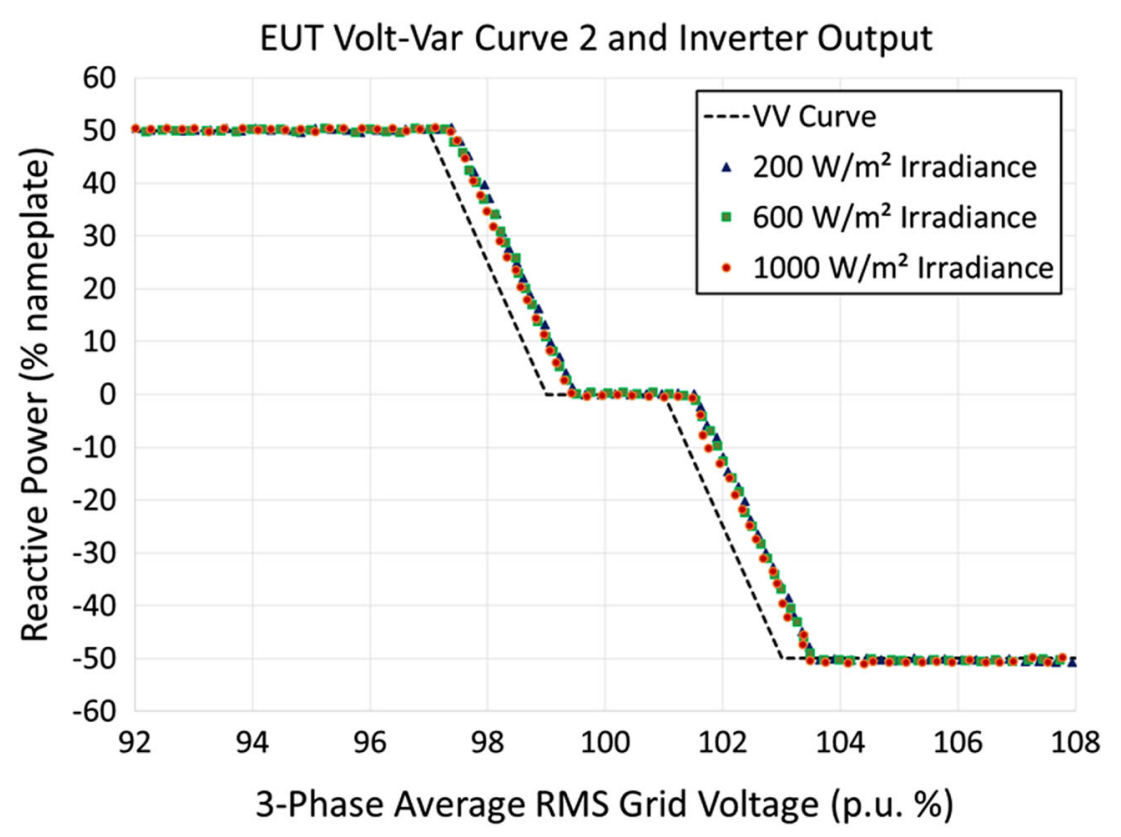

these functions can be programmed either as a parameterized curve or a pointwise function-as was the case for this EUT. To characterize the device FW behavior, the grid frequency was increased and decreased from nominal to $50.5,51.0$, and $52.0 \mathrm{~Hz}$ to determine the influence of hysteresis. The frequency profile was generated with $0.05 \mathrm{~Hz}$ steps and $1 \mathrm{sec}$ settling times. For this demonstration, the EUT power was measured through the frequency profile with the FW function enabled (blue trace) and disabled (orange trace). As shown in Fig. 12, the active power was reduced based on the FW curve when the function was enabled. The effect of the hysteresis is also shown in the figure with arrows indicating the direction of the frequency change.

\section{Discussion}

As shown by the CHIL experiments, test protocols can be quickly scripted and automated using the SunSpec SVP. The CHIL-SVP test configuration successfully enabled, adjusted, and executed six grid-support function certification tests using the AIT SGC as the EUT. The results show the effectiveness of using the CHIL approach to assist programmers in designing DER firmware to catch design
Fig. 12 FW behavior with the hysteresis loop indicated with arrows

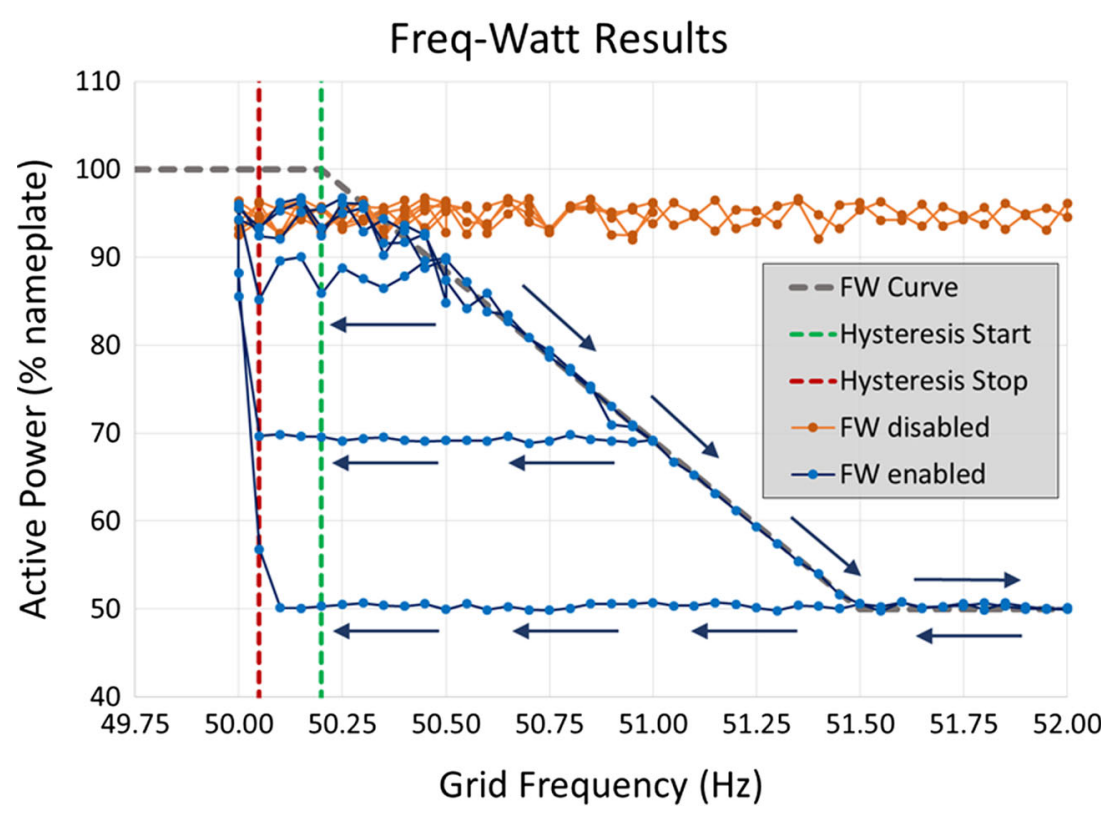


mistakes prior to deployment in hardware. The setup and operation of this type of testbed is significantly quicker and less expensive than running the experiments with power equipment.

The combination of the SVP and CHIL platforms are particularly useful for testing EUTs to certification protocols requiring a number of operating modes and/or settings. This capability reduces the burden on test engineers by automating the experiments and data collection, while still validating the functionality of the EUT early in the design process. Furthermore, coupling the interoperability (i.e., communications) tests with the electrical behavior testing is effective for certifying the equipment for both sets of requirements $[33,34]$. In this case, the EUT was shown to properly understand and update its behavior when receiving standardized SunSpec Modbus commands. Additional communications protocols such as IEEE 2030.5 (Smart Energy Profile 2.0), IEEE 1815 (DNP3), or IEC 61850 could be easily substituted in the SunSpec SVP to validate other communication protocols.

\section{Conclusions}

There is growing interest and research basis to conduct controller hardware-in-the-loop evaluations of smart grid devices. While this is not yet commonplace for equipment certification, it is particularly helpful for DER design processes, expedited firmware development, and interconnection and interoperability standards development. To demonstrate these advantages, Sandia, AIT, SunSpec, and Typhoon-HIL collaborated to create an automated smart inverter controller hardware-in-the-loop testbed. This platform and associated capabilities enables users to:

1. Complete certification experiments with limited power system hardware, e.g., no grid simulator, PV simulator, data acquisition system, load banks, etc.

2. Test large EUT controllers prior to integration with power equipment.

3. Accelerate the DER design cycle for interconnection and interoperability compliance.

4. Create optimized testing protocols for certification standards.

In this work, we have shown this CHIL capability through the demonstration and automation of advanced grid support tests with a $34.5 \mathrm{~kW}$ AIT SGC connected to a Typhoon HIL simulated power converter. We have demonstrated CHIL system response to connect/disconnect, active power curtailment, fixed $\mathrm{PF}$, reactive power, $\mathrm{VV}$, and $\mathrm{FW}$ advanced grid functions.

However, significant work remains to make this method of experimentation mainstream. As a first step, Sandia and SunSpec regularly release SVP test scripts in an online repository [35] for DER vendors, NRTLs, and other interested parties. Through the collaboration within ISGAN-SIRFN the partners also plan to actively contribute the work to national and international standards to introduce advanced CHIL methodologies as essential part of future DER certification testing.

Additionally, it is the intention of the team to provide the integrated CHIL certification platform to academic and research institutions around the world-especially in emerging economies where access to costly power equipment may be limited. In these markets, it will be particularly useful for Si-CHIL users to develop and refine interconnection and interoperability certification standards using the results from the integrated platform without the added time, electrical exposure, and expense of conducting full-scale power systems testing.

Acknowledgements Sandia National Laboratories is a multimission laboratory managed and operated by National Technology and Engineering Solutions of Sandia, LLC, a wholly owned subsidiary of Honeywell International, Inc., for the U.S. Department of Energy's National Nuclear Security Administration under contract DE-NA0003525.

The participation of AIT within ISGAN-SIRFN is funded in the frame of the IEA Research Cooperation program by the Austrian Ministry for Transport, Innovation and Technology under contract no. FFG 839566. The development of the AIT SGC was supported by the Austrian Ministry for Transport, Innovation and Technology (bmvit) and the Austrian Research Promotion Agency (FFG) under the "Energy Research Program 2015" in the SPONGE project (FFG no. 848915)

\section{References}

1. Bründlinger R, Strasser T, Lauss G, Hoke A, Chakraborty S, Martin G, Kroposki B, Johnson J, de Jong E (2015) Lab tests: verifying that smart grid power converters are truly smart. IEEE Power Energ Mag 13(2):30-42. https://doi.org/10.1109/ MPE.2014.2379935

2. Rosewater D, Johnson J, Verga M, Lazzari R, Messner C, Bründlinger R, Johannes K, Hashimoto J, Otani K (2015) International development of energy storage interoperability test protocols for renewable energy integration. EU PVSEC, Hamburg, 14-18 Sept

3. Bründlinger $\mathrm{R}$ Advanced smart inverter and DER functions requirements in latest European grid codes and future trends. Solar Canada, 8 Dec. 2015

4. Palmintier B, Broderick R, Mather B, Coddington M, Baker K, Ding F, Reno M, Lave M, Bharatkumar A (2016) On the path to sunshot: emerging issues and challenges in integrating solar with the distribution system. National Renewable Energy Laboratory, NREL/TP-5D00-65331

5. Johnson J, Neely J, Delhotal J, Lave M (2016) Photovoltaic frequency-watt curve design for frequency regulation and fast contingency reserves. IEEE J Photovoltaics 6(6):1611-1618

6. Seguin R, Woyak J, Costyk D, Hambrick J, Mather B (2016) Highpenetration $\mathrm{PV}$ integration handbook for distribution engineers. NREL Technical Report NREL/TP-5D00-63114 
7. Seal B (2014) Common functions for smart inverters, version 3. EPRI Report 3002002233, $21 \mathrm{Feb}$

8. IEC Technical Report IEC 61850-90-7 (2013) Communication networks and systems for power utility automation-part 90-7: object models for power converters in distributed energy resources (DER) Systems. Edition 1.0

9. IEC 61850-7-420 (2009) Communication networks and systems for power utility automation-part 7-420: basic communication structure-distributed energy resources logical nodes

10. IEEE Standard 1547-2003 (2003) Standard for interconnecting distributed resources with electric power systems

11. IEEE Standard 1547.1-2005 (2005) Standard for conformance test procedures for equipment interconnecting distributed resources with electric power systems

12. Underwriters Laboratories 1741 Ed. 2 (2010) Inverters, converters, controllers and interconnection system equipment for use with distributed energy resources

13. Johnson J, Bründlinger R, Urrego C, Alonso R (2014) Collaborative development of automated advanced interoperability certification test protocols for PV smart grid integration. EU PVSEC, Amsterdam, Netherlands, 22-26 Sept

14. Verga M, Lazzari R, Johnson J, Rosewater D, Messner C, Hashimoto J (2016) SIRFN draft test protocols for advanced battery energy storage system interoperability functions. ISGAN Annex \#5 Discussion Paper

15. SunSpec Alliance. SunSpec System Validation Platform (SVP), software, http://sunspec.org/sunspec-svp/

16. Johnson J, Fox B (2014) Automating the Sandia advanced interoperability test protocols. In: 40th IEEE PVSC, Denver, 8-13 June

17. Miletic Z. AIT Smart Grid Converter (SGC) control platform: using controller hardware in the loop for the AIT Smart Grid Converter (SGC) control platform, Webinar. http://info.typhoon-hil.com/recorded-webinar-ait-smart-grid-con verter-control-platform-and-controller-hardware-in-the-loop

18. Bründlinger R. SunSpec and AIT Smart Grid Converter (SGC) control platform: using controller hardware in the loop to implement SunSpec protocols in the AIT SGC control platform, Webinar

19. Bründlinger R, Ablinger R, Miletic Z (2016) AIT Smart Grid Converter (SGC) controller featuring SunSpec protocol support utilizing Hardware-in-the-Loop (HIL) technology, SunSpec Meeting, September 13

20. Johnson J, Ablinger R, Bruendlinger R, Fox B, Flicker $\mathrm{J}$ (2017) Interconnection Standard Grid-Support Function Evaluations using an Automated Hardware-in-the-Loop Testbed, IEEE PVSC, Washington, DC, 25-30 June

21. Steurer MM et al (2016) Multifunctional megawatt-scale medium voltage DC test bed based on modular multilevel converter technology. IEEE Trans Transp Electrification 2(4):597-606. https://doi.org/10.1109/TTE.2016.2582561
22. Maitra A et al (2017) Microgrid controllers: expanding their role and evaluating their performance. IEEE Power Energ Mag 15(4):41-49. https://doi.org/10.1109/MPE.2017.2690519

23. Panwar M, Lundstrom B, Langston J, Suryanarayanan S, Chakraborty S (2013) An overview of real time hardware-in-theloop capabilities in digital simulation for electric microgrids. In: 2013 North American Power Symposium (NAPS), Manhattan, pp 1-6. https://doi.org/10.1109/NAPS.2013.6666861

24. Lundstrom B, Chakraborty S, Lauss G, Bründlinger R, Conklin R (2016) Evaluation of system-integrated smart grid devices using software- and hardware-in-the-loop. In: 2016 IEEE power \& energy society innovative smart grid technologies conference (ISGT), Minneapolis, pp 1-5. https://doi.org/10.1109/ ISGT.2016.7781181

25. Lundstrom B, Shirazi M, Coddington M, Kroposki B (2013) An advanced platform for development and evaluation of grid interconnection systems using hardware-in-the-loop: part IIIgrid interconnection system evaluator. In: 2013 IEEE green technologies conference (GreenTech), Denver, pp 392-399. https://doi.org/10.1109/GreenTech.2013.67

26. IEEE Project. P2030.8 - standard for the testing of microgrid controllers. https://standards.ieee.org/develop/project/2030.8.html

27. IEEE Project. P2004-hardware-in-the-Loop (HIL) simulation based testing of electric power apparatus and controls. https:// standards.ieee.org/develop/project/2004.html

28. Hoke A, Chakraborty S, Basso T, Coddington M (2014) Beta test plan for advanced inverters interconnecting distributed resources with electric power systems. NREL Technical Report NREL/TP5D00-60931

29. Electric Power Research Institute (2017) IEEE 1547-new interconnection requirements for distributed energy resources: fact sheet. Report 3002011346

30. SunSpec Alliance. SunSpec Dashboard, software, http://sunspec. org/sunspec-dashboard/

31. Johnson J, Schenkman B, Ellis A, Quiroz J, Lenox C (2012) Initial operating experience of the 1.2-MW La Ola photovoltaic system. In: 38th IEEE PVSC, Austin, TX, 4 June

32. VDE Reference (2008) VDE-AR-N 4105 power generation systems connected to the low voltage distribution network -technical minimum requirements for the connection to and parallel operation with low voltage distribution networks

33. Gonzalez J, Johnson S, Ralph ME, Ellis A, Broderick R (2013) Test protocols for advanced inverter interoperability functionsmain document. Sandia Technical Report SAND2013-9880

34. Gonzalez J, Johnson S, Ralph ME, Ellis A, Broderick R (2013) Test protocols for advanced inverter interoperability functionsappendices. Sandia Technical Report SAND2013-9875

35. System Validation Platform Github Repository, https://github. com/sunspec/svp_directories 